\title{
Ballistic thermoelectric properties of monolayer semiconducting transition metal dichalcogenides and oxides
}

\author{
G. Özbal $\odot,{ }^{1}$ R. T. Senger, ${ }^{1,2}$ C. Sevik,${ }^{3}$ and H. Sevinçli ${ }^{4, *}$ \\ ${ }^{1}$ Department of Physics, Izmir Institute of Technology, 35430 Izmir, Turkey \\ ${ }^{2}$ ICTP-ECAR Eurasian Center for Advanced Research, Izmir Institute of Technology, 35430 Izmir, Turkey \\ ${ }^{3}$ Department of Mechanical Engineering, Faculty of Engineering, Eskişehir Technical University, Eskişehir, TR 26555, Turkey \\ ${ }^{4}$ Department of Materials Science and Engineering, Izmir Institute of Technology, 35430 Izmir, Turkey
}

(Received 29 January 2019; revised manuscript received 17 May 2019; published 12 August 2019)

\begin{abstract}
Combining first-principles calculations with Landauer-Büttiker formalism, ballistic thermoelectric transport properties of semiconducting two-dimensional transition metal dichalcogenides (TMDs) and oxides (TMOs) (namely $\mathrm{MX}_{2}$ with $\mathrm{M}=\mathrm{Cr}$, Mo, W, Ti, Zr, Hf; X $=\mathrm{O}, \mathrm{S}, \mathrm{Se}, \mathrm{Te}$ ) are investigated in their $2 \mathrm{H}$ and $1 \mathrm{~T}$ phases. Having computed structural, as well as ballistic electronic and phononic transport properties for all structures, we report the thermoelectric properties of the semiconducting ones. We find that $2 \mathrm{H}$ phases of four of the studied structures have very promising thermoelectric properties, unlike their $1 \mathrm{~T}$ phases. The maximum room temperature $p$-type thermoelectric figure of merit $(Z T)$ of 1.57 is obtained for $2 \mathrm{H}-\mathrm{HfSe}_{2}$, which can be as high as 3.30 at $T=800 \mathrm{~K}$. Additionally, $2 \mathrm{H}-\mathrm{ZrSe}_{2}, 2 \mathrm{H}-\mathrm{ZrTe}_{2}$, and $2 \mathrm{H}-\mathrm{HfS}_{2}$ have considerable $\mathrm{ZT}$ values (both $n$ and $p$-type), that are above 1 at room temperature. The $1 \mathrm{~T}$ phases of $\mathrm{Zr}$ and Hf-based oxides possess relatively high power factors, however their high lattice thermal conductance values limit their $Z T$ values to below 1 at room temperature.
\end{abstract}

DOI: 10.1103/PhysRevB.100.085415

\section{INTRODUCTION}

Thermoelectric (TE) materials make it possible to drive electric currents using temperature gradients, and conversely cooling of a system just by using a voltage difference, namely the Seebeck and Peltier effects, respectively. The performance of TE conversion is quantified by the dimensionless figure of merit $Z T$, which includes strongly interrelated electronic and thermal transport properties. Because of this interrelation among Seebeck coefficient $(S)$, electrical conductance $(G)$, and thermal conductance $(\kappa)$, significant enhancement of $Z T$ is an extremely difficult task. Therefore improvement of TE efficiency has been relatively slow, and typical $Z T$ values do not exceed 1 for bulk materials [1]. With the advances in the production of low-dimensional structures, a new quest for high performance TE materials gained acceleration [2-7]. The advent of atomically thin graphene provided a new platform to study transport and thermoelectric properties in two and one dimensions (2D and 1D) [8-10]. However, the absence of an electronic band gap in 2D graphene and ultrahigh thermal conductivity suppress its thermoelectric efficiency [11-15]. Still, there are numerous proposals to enhance the TE performance of graphene [9,16-26]. A more recent family of 2D materials, semiconducting TMDs and TMOs attracted attention due to the wide range of band gaps and lower lattice thermal conductivities. One of the most detailed studies on stability, electronic, mechanical, and magnetic analysis of single layer TMDs and TMOs belongs to Ataca et al. [27]. The majority of the theoretical studies have been devoted towards

*haldunsevincli@iyte.edu.tr
$\mathrm{MX}_{2}(\mathrm{M}=\mathrm{Mo}, \mathrm{W} ; \mathrm{X}=\mathrm{S}, \mathrm{Se})$ monolayers [28-36], few layers [37-42], hybrid nanoribbons [43,44], or heterostructures [45]. Phonon engineering [46], band structure engineering [47], and strain engineering [48-52] approaches were also employed extensively with the aim of improving thermoelectric performance of $\mathrm{MX}_{2}$ structures. In addition to Mo and $\mathrm{W}$ based compounds, there are also a few studies $\mathrm{Zr}$ and $\mathrm{Hf}$ based TMDs in their 1T phases [53-57]. However, a comprehensive study on thermoelectric properties of pristine TMD/TMOs, specifically the $2 \mathrm{H}$ phase of $\mathrm{Ti}, \mathrm{Zr}$, and $\mathrm{Hf}$ based structures, is still lacking. Here, we focus on expanding the library of 2D TMD/TMO candidates starting with an investigation of their ballistic properties. Electronic transport, thermal transport, and thermoelectric properties of 26 dynamically stable semiconducting TMDs/TMOs are explored. Structural parameters are computed for obtaining accurate electronic band structures and vibrational spectra based on ab initio calculations. Thermoelectric coefficients are computed by combining first-principles calculations and Landauer-Büttiker formalism. Also band gap corrections are performed using hybrid HSE06 functionals when necessary.

\section{METHODS}

The geometrical optimization and electronic structure calculations are performed using density functional theory (DFT) using plane-wave basis sets [58] by employing projector augmented wave (PAW) potentials [59]. The exchangecorrelation potential has been approximated by generalized gradient approximation (GGA) using Perdew-BurkeErnzerhof (PBE) functionals [60]. The plane-wave cutoff energies are found to be in the range from 250 to $500 \mathrm{eV}$ with 
convergence tests for each structure. The irreducible Brillouin zone is sampled using the Monkhorst-Pack scheme with grid sizes of $n \times n \times 1 \quad(n=5-15)$ according to the convergence tests [61]. The convergence thresholds for ionic and electronic relaxations are set to $10^{-3} \mathrm{eV} / \AA$ and $10^{-6} \mathrm{eV}$, respectively. During the geometry optimization process, cell shape and volume are preserved. The vacuum spacing is set to $15 \AA$ to avoid any spurious interactions between layers. In order to correct the band gap values Heyd-Scuseria-Ernzerhorf (HSE06) [62] hybrid functionals are used for selected five $\mathrm{MX}_{2}$ structures, where 0.25 exact Hartree-Fock and 0.75 PBE exchange mixing and the screening parameter of $0.2 \AA^{-1}$ are used. The calculations are performed non-spin-polarized and spin-orbit interactions are not taken into consideration. The interatomic force constants (IFCs) are obtained by employing density functional perturbation theory (DFPT) [63]. Phonon band structure and heat capacity calculations are performed by using the PHONOPY package [64].

The cohesive energy $\left(\mathrm{E}_{c}\right)$ per atom is computed as

$$
E_{\text {coh }}=\left(n_{\mathrm{X}} E_{\mathrm{X}}+n_{\mathrm{M}} E_{\mathrm{M}}-E_{\mathrm{MX}_{2}}\right) /\left(n_{\mathrm{X}}+n_{\mathrm{M}}\right) .
$$

Here, $n_{X(M)}$ denotes the number of chalcogen (transition metal) atoms in the unit cell. $E_{X(M)}$ is the energy of the isolated single atoms, and $E_{M X_{2}}$ is the total energy of the $\mathrm{MX}_{2}$ monolayer. In order to gain an understanding on bond characteristics, charge transfer calculations are conducted by using the Bader method [65]. The percent ionic character (\%IC) of metal and chalcogen/oxygen atoms can be calculated roughly as [66]

$$
\% I C=\left\{1-\exp \left[-0.25\left(X_{\mathrm{A}}-X_{\mathrm{B}}\right)^{2}\right]\right\} \times 100
$$

where $X_{\mathrm{A}}$ and $X_{\mathrm{B}}$ are electronegativities of the constituent atoms.

Electronic transmission spectrum is given by the number of transmission channels in the ballistic limit. Since each studied structure exhibits hexagonal symmetry, their transmission spectra are isotropic. Therefore both transmission spectra and thermoelectric coefficients are given along one direction. Dense $k$-point meshes $200 \times 200 \times 1$ and $100 \times$ $100 \times 1$ are used in calculating transmission spectra using PBE and HSE06 functionals, respectively.

Derivation of the electronic coefficients is performed by using $[67,68]$

$$
L_{n}(\mu, T)=-\frac{2}{h} \int d E \tau_{e l}(E)(E-\mu)^{n} \frac{\partial f_{\mathrm{FD}}(E, \mu, T)}{\partial E},
$$

with $n$ being an integer, $\tau_{e l}(E)$ the electronic transmission spectrum, and $f_{\mathrm{FD}}(E, \mu, T)$ the Fermi-Dirac distribution function at temperature $T$ and chemical potential $\mu$. Using $L_{n}$, one can express the electrical conductance $(G)$, Seebeck coefficient $(S)$, and the electrical part of the thermal conductance $\left(\kappa_{\mathrm{el}}\right)$ as $G=e^{2} L_{0}, S=\left(L_{1} / L_{0}\right) / e T$, and $\kappa_{\mathrm{el}}=\left(L_{2}-\right.$ $\left.L_{1}^{2} / L_{0}\right) / T$, respectively.

Phonon thermal conductance is calculated using Landauer formalism $[69,70]$,

$$
\kappa_{\mathrm{ph}}=\frac{1}{2 \pi} \int d \omega \hbar \omega \tau_{p h}(\omega) \frac{\partial f_{\mathrm{BE}}(\omega, T)}{\partial T},
$$

where $\omega$ is the vibrational frequency, $f_{\mathrm{BE}}$ stands for BoseEinstein distribution function, and $\tau_{p h}(\omega)$ is the phonon

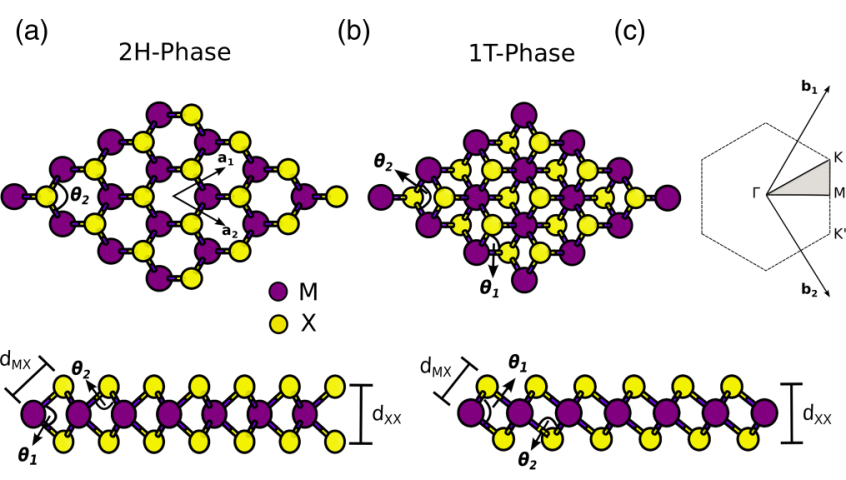

FIG. 1. Crystal structures of TMDs and TMOs. The $2 \mathrm{H}$ phase (a) and the $1 \mathrm{~T}$ phase (b). The Brillouin zone, reciprocal lattice vectors, and the high symmetry points are given in (c).

transmission spectrum obtained from counting phonon modes with an average of $200 q$ points in the transverse direction. After computing the electronic and phononic contributions to the transport, the dimensionless thermoelectric figure of merit is obtained using

$$
Z T=S^{2} G T /\left(\kappa_{\mathrm{el}}+\kappa_{\mathrm{ph}}\right) .
$$

\section{STRUCTURAL AND ELECTRONIC PROPERTIES}

$\mathrm{MX}_{2}$ monolayers consist of three atomic layers in the sequence of $\mathrm{X}-\mathrm{M}-\mathrm{X}$. These sublayers are arranged in the well-known two phases (polymorphs): trigonal prismatic $2 \mathrm{H}$ which is a member of $P \overline{6} m 2\left(D_{3 h}\right)$ symmetry group and octahedral $1 \mathrm{~T}$ which belongs to the $P \overline{3} m 1\left(D_{3 d}\right)$. Schematic representation of $2 \mathrm{H}$ and $1 \mathrm{~T}$ phases from top and side views are shown in Figs. 1(a) and 1(b), respectively, and their first Brillouin zone is given in Fig. 1(c).

We first perform geometry optimizations and check the dynamical stabilities of the TMD/TMO monolayers by checking whether all vibrational frequencies are real and positive. While all $2 \mathrm{H}$ structures group-VIB $(\mathrm{Cr}, \mathrm{Mo}, \mathrm{W})$ TMDs/TMOs are dynamically stable semiconductors, their $1 \mathrm{~T}$ phases are unstable. Their distorted $1 \mathrm{~T}_{d}$ phases are dynamically stable, but all are metallic and therefore they are not within the scope of this study. On the other hand, both $2 \mathrm{H}$ and $1 \mathrm{~T}$ phases of group IVB (Ti, Zr, Hf) TMDs/TMOs are dynamically stable and semiconducting. The structural parameters which determine the geometry are tabulated in Table I. The obtained parameters are in good agreement with the literature [71].

For a given phase, $d_{\mathrm{MX}}$ increases with increasing $a$. However, different phases $(2 \mathrm{H}$ and $1 \mathrm{~T})$ of a given $\mathrm{MX}_{2}\left(\mathrm{ZrSe}_{2}\right.$, $\mathrm{HfS}_{2}$, and $\mathrm{HfSe}_{2}$ ) follow an opposite trend. For example, the $1 \mathrm{~T}$ phase of $\mathrm{ZrSe}_{2}$ has larger $a$ than its $2 \mathrm{H}$ phase but smaller $d_{\mathrm{MX}}$ and $h$. For these structures, $a$ is always larger for the 1T phase, whereas $d_{\mathrm{MX}}$ is reduced by $\sim 0.7 \%$ and $h$ is reduced by $\sim 6-7 \%$. Bond angles $\theta_{1}$ and $\theta_{2}$ follow opposite trends. Comparing two $\mathrm{MX}_{2}$ structures, one observes that the structure with larger $\theta_{1}$ has smaller $\theta_{2}$. Also $\theta_{1}$ of the $1 \mathrm{~T}$ phase of a given structure is always larger than that in the $2 \mathrm{H}$ phase.

For compounds of the same phase, $E_{\mathrm{coh}}$ decreases with increasing $a$ as expected. A comparison of $2 \mathrm{H}$ and 1T phases of the same TMD reveal that $E_{\mathrm{coh}}$ is always larger for the 1T 
TABLE I. Structural and electronic properties of semiconducting TMDs and TMOs, which are dynamically stable. The lattice parameter of the unit cell $(a)$, bond lengths $\left(d_{\mathrm{MX}}\right)$, layer heights $(h)$, bond angles $\left(\theta_{1}, \theta_{2}\right)$, band gap $\left(E_{\mathrm{g}}^{\mathrm{PBE}}\right)$, cohesive energy $\left(E_{\mathrm{coh}}\right)$, transferred charge to $\mathrm{X}\left(\rho_{M}\right)$, charge received by $\mathrm{X}\left(\rho_{X}\right)$, and the fractional ionic character (FIC), respectively. Bond lengths and angles are shown in Fig. 1 and electronic band structures are illustrated in Fig. S2 [93].

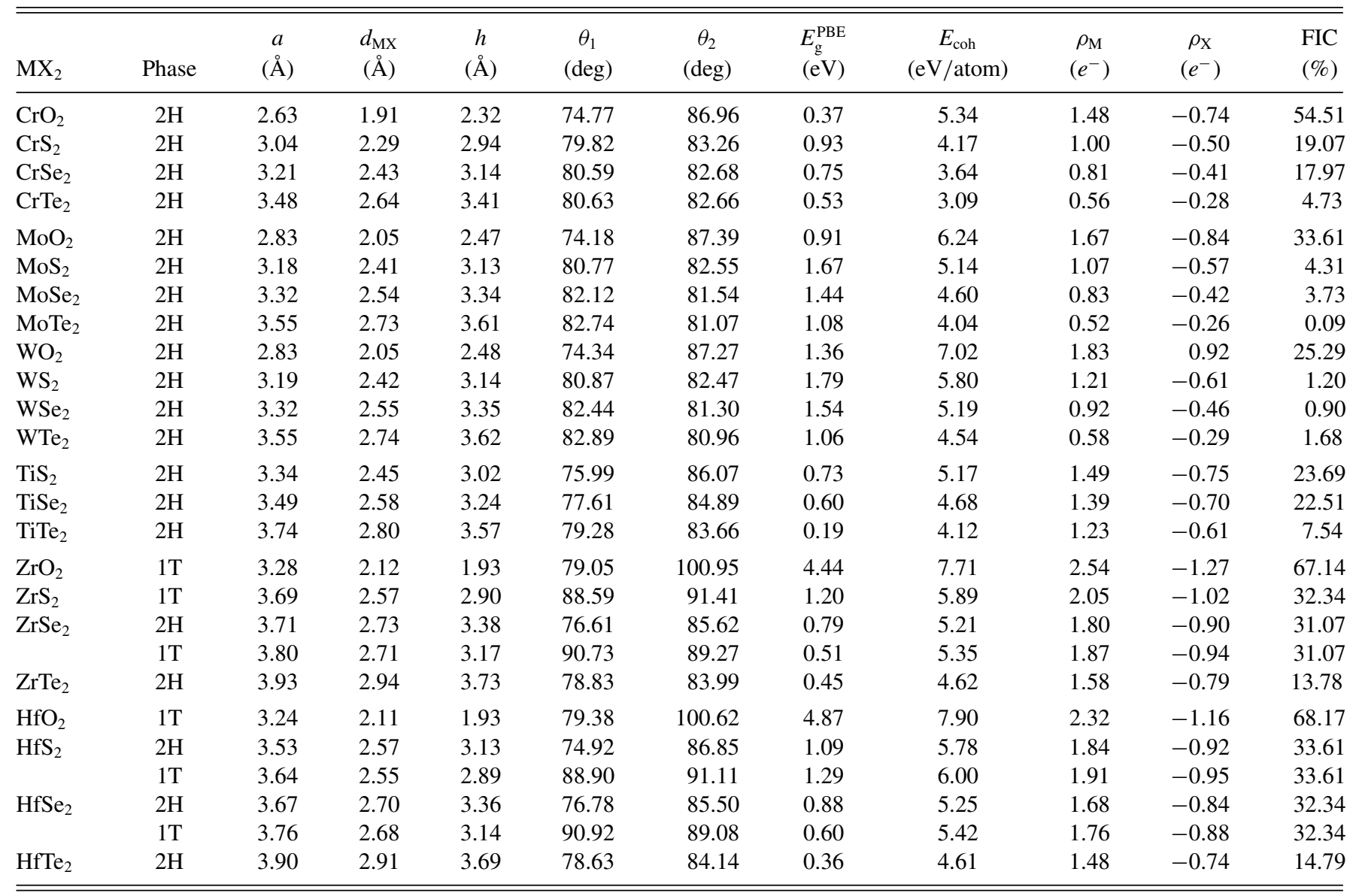

phase. That is to say, $1 \mathrm{~T}$ phases of studied $\mathrm{Zr}$ and $\mathrm{Hf}$ based compounds are energetically more stable, in agreement with previous studies [72-76]. According to Table I, TMOs exhibit the highest ionic character in general, which is because of the largest charge transfer between the transition metal and the oxygen atoms. 1T- $\mathrm{HfO}_{2}$, which has the highest cohesive energy and the widest electronic band gap shows the highest ionic character, whereas $2 \mathrm{H}-\mathrm{MoTe}_{2}$ possesses fractional covalent character.

Electronic band diagrams of the investigated structures are plotted in the Supplemental Material (see Fig. S2) [93]. The $2 \mathrm{H}$ phases of group-VIB dichalcogenides are direct band gap semiconductors, whereas their oxides have indirect band gaps. Group-IVB dichalcogenides and oxides are all indirect semiconductors. Electronic band gaps ranging between $0.19 \mathrm{eV}$ and $4.87 \mathrm{eV}$ are obtained with PBE functionals. HSE06 calculations are performed for materials which have a $E_{\mathrm{g}}^{\mathrm{PBE}}$ less than $0.5 \mathrm{eV}$. This is because the main effect of the hybrid functional to the band structure is to increase the band gap with changing the band dispersions only slightly. When the band gap of a structure is less than $10 k_{B} T$, simultaneous contribution from the holes in the valence band and electrons in the conduction band suppresses the Seebeck coefficient [77-81]. The effect of hybrid functionals on electronic transport and thermoelectric properties will be discussed in more detail later. The electronic band diagrams of $2 \mathrm{H}-\mathrm{CrO}_{2}, 2 \mathrm{H}-\mathrm{TiTe}_{2}$, $2 \mathrm{H}-\mathrm{ZrTe}_{2}$, and $2 \mathrm{H}-\mathrm{HfTe} \mathrm{T}_{2}$ within PBE+HSE06 functionals are presented in Fig. 2. Band gap values are increased to 0.90, 0.97, 1.05, and $0.93 \mathrm{eV}$ for $\mathrm{CrO}_{2}, \mathrm{TiTe}_{2}, \mathrm{ZrTe}_{2}$, and $\mathrm{HfTe}_{2}$, respectively.

\section{VIBRATIONAL PROPERTIES}

It is necessary to check whether imaginary or negative frequencies exist in phonon dispersions to check the dynamical stabilities of the structures. We note that dynamical stability is a necessary condition but not conclusive for experimental realization. When both $2 \mathrm{H}$ and $1 \mathrm{~T}$ phases of $\mathrm{MX}_{2}$ monolayers are considered, 30 structures are found to be dynamically stable. Four of these structures are excluded in this study as they are either metallic or semi-metallic. Phonon spectra of the remaining 26 structures are given in the Supplemental Material (see Fig. S1) [93]. A cautionary note is in order here. Smearing is a computational tool that smoothens the Fermi distribution function around the Fermi energy. It is a necessary ingredient in DFT calculations. Even graphene can be found dynamically unstable if appropriate smearing is not used. There does not exist a recipe for determining the smearing method or value. There are a few methods to implement smearing, most of them without a clear 

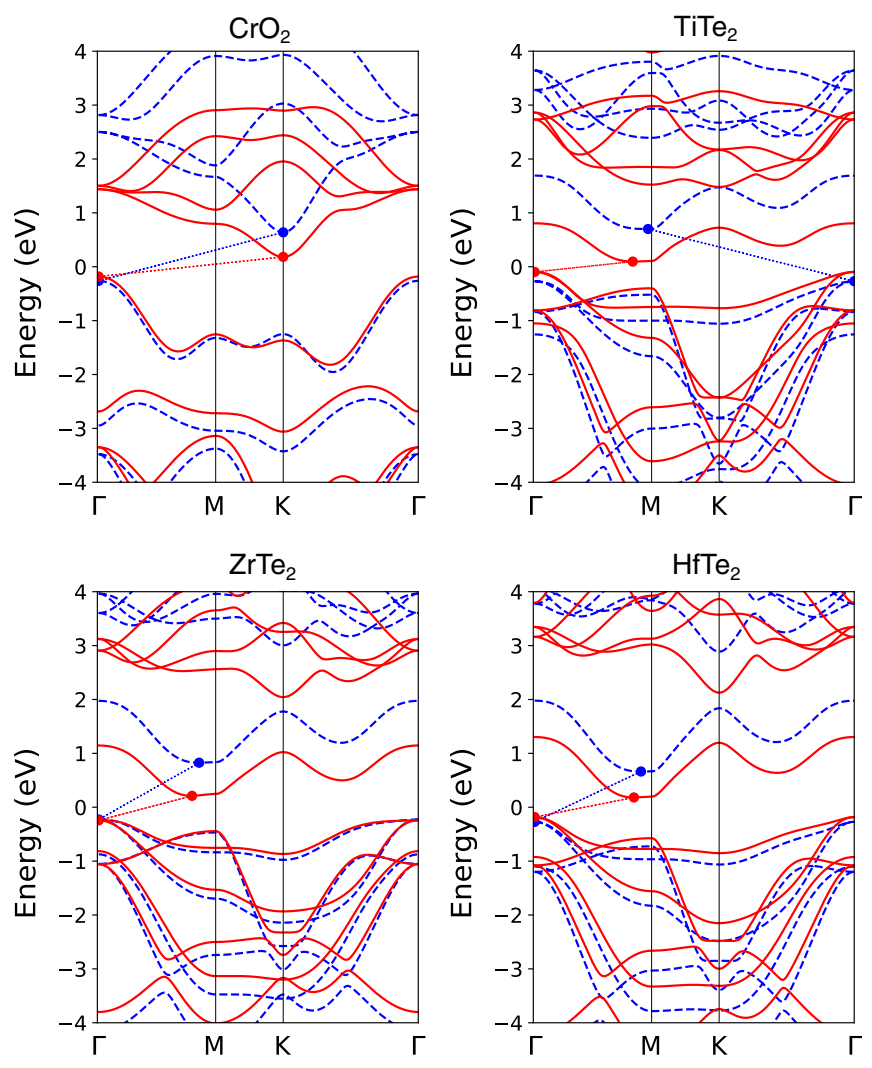

FIG. 2. Calculated electronic band structures of selected 2H-MX $\mathrm{MX}_{2}$ compounds; $\mathrm{CrO}_{2}, \mathrm{TiTe}_{2}, \mathrm{ZrTe}_{2}, \mathrm{HfTe}_{2}$ based on PBE (red solid line) and PBE+HSE (blue dashed line) functional. Fermi level is set to zero for all subfigures.

physical meaning. Fermi-Dirac smearing, on the other hand, is interpreted as electronic temperature. Being only applied on electrons and not on ions, it should be not confused with real temperature. Still, Fermi-Dirac smearing is used to identify temperature dependent stabilization in certain cases [82-84]. In this work, we implement Fermi-Dirac smearing and scan possible smearing values systematically.

For lower values of smearing $(\sigma=0.05 \mathrm{eV}) 2 \mathrm{H}-\mathrm{TiS}_{2}$, $2 \mathrm{H}-\mathrm{ZrSe}_{2}$, and $2 \mathrm{H}-\mathrm{HfS}_{2}$ out-of-plane ZA mode possess negative frequencies around the high symmetry points, whereas $2 \mathrm{H}-\mathrm{HfSe}_{2}$ has negative frequencies ony around the $K$ point. When the smearing is increased $\left(\sigma=0.4 \mathrm{eV}\right.$ for $2 \mathrm{H}-\mathrm{HfSe}_{2}$ and $\sigma=0.5 \mathrm{eV}$ for $2 \mathrm{H}-\mathrm{TiS}_{2}, 2 \mathrm{H}-\mathrm{ZrSe}_{2}, 2 \mathrm{H}-\mathrm{HfS}_{2}$ ) all phonons frequencies are found positive. These $\sigma$ values are in the same range with those used in the literature $[82,85,86]$.

The phonon band gap, which separates the acoustic modes from the six optical branches, decreases with decreasing mass difference between the constituent elements of $\mathrm{MX}_{2}$ compounds. The acoustic bandwidths become narrower with increasing average mass, whereas all bands are pushed towards lower frequencies with increasing total mass of the compounds. In previous studies, in which phonon-phonon scattering was taken into account, the scatterings were limited when a band gap is present. Therefore the absence of a phonon band gap was found helpful to reduce lattice thermal conductivity. Conversely, in the ballistic regime, the presence of a phonon band gap reduces lattice thermal conductivity,
TABLE II. Phonon thermal conductance values for various temperatures.

\begin{tabular}{|c|c|c|c|c|}
\hline \multicolumn{5}{|c|}{$\kappa_{p h}(\mathrm{nW} / \mathrm{K} / \mathrm{nm})$} \\
\hline$M X_{2}$ & Phase & $300 \mathrm{~K}$ & $500 \mathrm{~K}$ & $800 \mathrm{~K}$ \\
\hline $\mathrm{CrO}_{2}$ & $2 \mathrm{H}$ & 2.09 & 2.49 & 2.66 \\
\hline $\mathrm{CrS}_{2}$ & $2 \mathrm{H}$ & 1.24 & 1.34 & 1.37 \\
\hline $\mathrm{CrSe}_{2}$ & $2 \mathrm{H}$ & 0.83 & 0.87 & 0.88 \\
\hline $\mathrm{CrTe}_{2}$ & $2 \mathrm{H}$ & 0.60 & 0.62 & 0.63 \\
\hline $\mathrm{MoO}_{2}$ & $2 \mathrm{H}$ & 1.63 & 1.89 & 2.00 \\
\hline $\mathrm{MoS}_{2}$ & $2 \mathrm{H}$ & 1.03 & 1.10 & 1.13 \\
\hline $\mathrm{MoSe}_{2}$ & $2 \mathrm{H}$ & 0.72 & 0.75 & 0.76 \\
\hline $\mathrm{MoTe}_{2}$ & $2 \mathrm{H}$ & 0.54 & 0.55 & 0.55 \\
\hline $\mathrm{WO}_{2}$ & $2 \mathrm{H}$ & 1.29 & 1.48 & 1.56 \\
\hline $\mathrm{WS}_{2}$ & $2 \mathrm{H}$ & 0.83 & 0.89 & 0.91 \\
\hline $\mathrm{WSe}_{2}$ & $2 \mathrm{H}$ & 0.66 & 0.68 & 0.68 \\
\hline $\mathrm{WTe}_{2}$ & $2 \mathrm{H}$ & 0.50 & 0.51 & 0.51 \\
\hline $\mathrm{TiS}_{2}$ & $2 \mathrm{H}$ & 0.95 & 1.00 & 1.02 \\
\hline $\mathrm{TiSe}_{2}$ & $2 \mathrm{H}$ & 0.95 & 0.99 & 1.00 \\
\hline $\mathrm{TiTe}_{2}$ & $2 \mathrm{H}$ & 0.70 & 0.72 & 0.73 \\
\hline $\mathrm{ZrO}_{2}$ & $1 \mathrm{~T}$ & 1.45 & 1.71 & 1.81 \\
\hline $\mathrm{ZrS}_{2}$ & $1 \mathrm{~T}$ & 0.83 & 0.87 & 0.89 \\
\hline \multirow[t]{2}{*}{$\mathrm{ZrSe}_{2}$} & $2 \mathrm{H}$ & 0.54 & 0.55 & 0.56 \\
\hline & $1 \mathrm{~T}$ & 0.71 & 0.72 & 0.73 \\
\hline $\mathrm{ZrTe}_{2}$ & $2 \mathrm{H}$ & 0.55 & 0.56 & 0.56 \\
\hline $\mathrm{HfO}_{2}$ & $1 \mathrm{~T}$ & 1.28 & 1.50 & 1.60 \\
\hline \multirow[t]{2}{*}{$\mathrm{HfS}_{2}$} & $2 \mathrm{H}$ & 0.65 & 0.67 & 0.68 \\
\hline & $1 \mathrm{~T}$ & 0.71 & 0.74 & 0.75 \\
\hline \multirow[t]{2}{*}{$\mathrm{HfSe}_{2}$} & $2 \mathrm{H}$ & 0.51 & 0.51 & 0.52 \\
\hline & $1 \mathrm{~T}$ & 0.59 & 0.61 & 0.61 \\
\hline $\mathrm{HfTe}_{2}$ & $2 \mathrm{H}$ & 0.48 & 0.49 & 0.49 \\
\hline
\end{tabular}

simply because there is no transmission within gap. Briefly, both a wide phonon band gap and reduced phonon frequencies decrease thermal conductance and enhance the TE performance. Thermal conductance of the investigated TMD/TMOs at various temperatures are listed in Table II. $2 \mathrm{H}-\mathrm{CrO}_{2}$ is composed of the lightest atoms in the group, hence has the largest phonon thermal conductance at all temperatures. As temperature increases thermal conductance of $\mathrm{CrO}_{2}$ increases considerably. A similar trend appears for all the TMOs, which is associated with the relatively higher $\omega_{\max }$ values, because of oxygen being the lightest element in group VIA. As will be discussed later, $2 \mathrm{H}$ phases of $\mathrm{ZrSe}_{2}, \mathrm{ZrTe}_{2}, \mathrm{HfS}_{2}$, and $\mathrm{HfSe}_{2}$ are found to be both $n$ - and $p$-type promising thermoelectric candidates. In order to clarify influence of thermal properties on thermoelectric performance, vibrational spectra and transport properties are presented in Fig. 3, only for these four materials.

In a previous study, which investigated the thermal conductivities of $2 \mathrm{H}$ group-VIB TMD family by using the Boltzmann transport equation [87], it was found that the thermal conductivities of sulfides $\left(\mathrm{MS}_{2}\right)$ and selenides $\left(\mathrm{MSe}_{2}\right)$ increase as $\mathrm{M}$ changes from $\mathrm{Cr}$ to $\mathrm{Mo}$, and from Mo to $\mathrm{W}$, due to the rapid increase in the phonon relaxation time. In contrast to this, we find that $\kappa_{\text {ph }}$ decreases as $\mathrm{M}$ changes from $\mathrm{Cr}$ to Mo to $\mathrm{W}$ at the ballistic limit, because of increasing atomic masses. This inverse behavior is validated with the calculation 

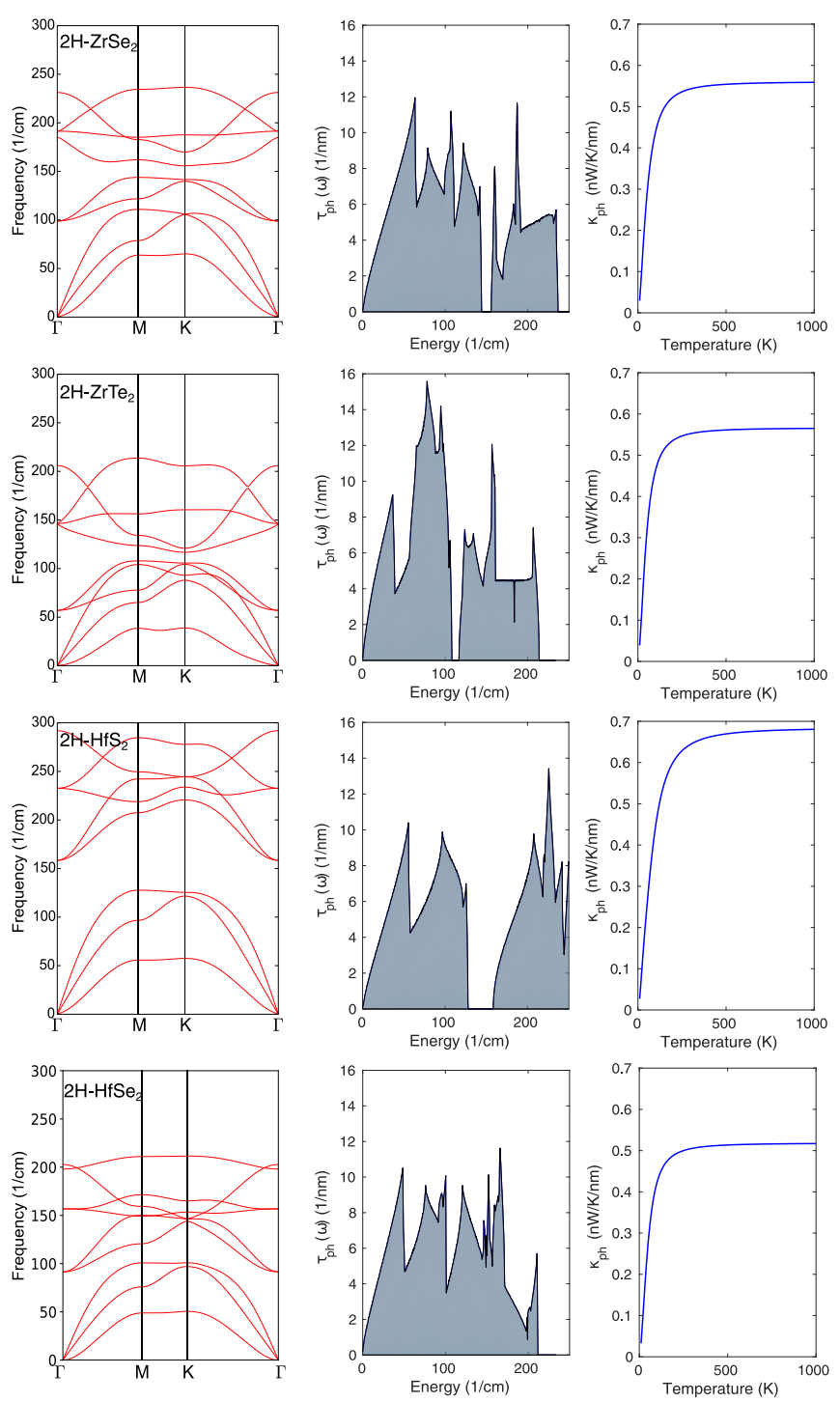

FIG. 3. Phonon dispersion relations, phonon transmission spectra, and phonon thermal conductance as a function of temperature of $\mathrm{ZrSe}_{2}, \mathrm{ZrTe}_{2}, \mathrm{HfS}_{2}$, and $\mathrm{HfSe}_{2}$ are shown, respectively.

of correlation between average atomic mass of the unit cell and phonon thermal conductance, where $\% 80, \% 78$, and $\% 77$ inverse correlations are found for $300 \mathrm{~K}, 500 \mathrm{~K}$, and $800 \mathrm{~K}$, respectively. In addition, it is found that the correlation between $m$ and $\kappa_{\mathrm{ph}}$ does not change at even higher temperatures.

We also note that in a recent theoretical study the ballistic thermal conductance value of $\mathrm{MoS}_{2}$ was reported as $1.06 \mathrm{nW} / \mathrm{K}$ for a sample having a width of $1.27 \mathrm{~nm}$ [88]. The corresponding thermal conductance per width value $(0.84$ $\mathrm{nW} \mathrm{K} \mathrm{K}^{-1} \mathrm{~nm}^{-1}$ ) is considerably less than our present result $\left(1.03 \mathrm{nW} \mathrm{K}^{-1} \mathrm{~nm}^{-1}\right)$. The disagreement is because we employ a fine sampling of the $k$ points in the transmission spectrum, whereas Cai et al. uses only the $\Gamma$ point. Hence they find a stepwise transmission spectrum like in a onedimensional system, which overestimates the contributions from low energies.
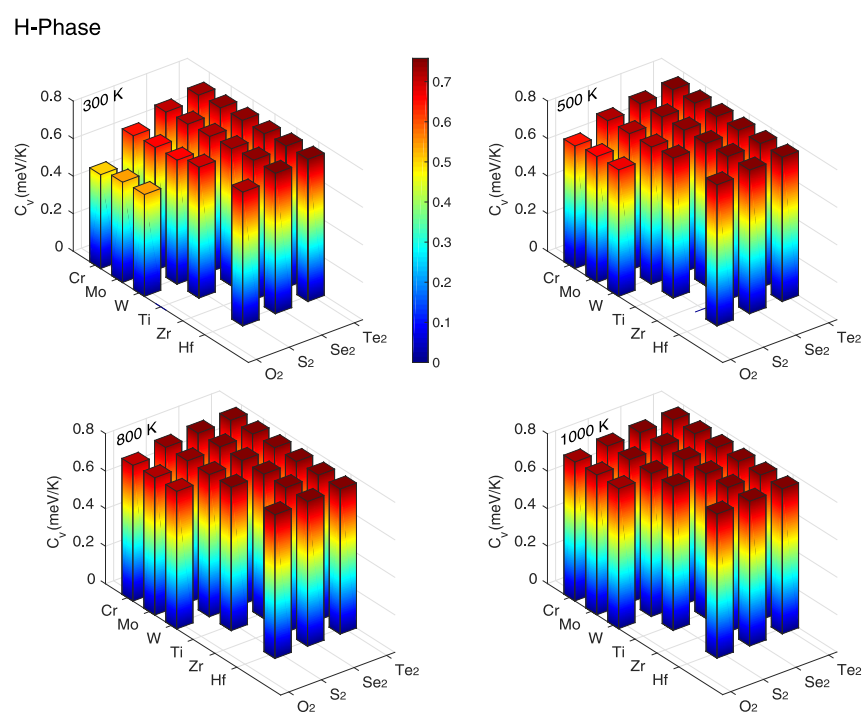

FIG. 4. Heat capacities at various temperatures from $300 \mathrm{~K}$ to $1000 \mathrm{~K}$ are shown for the semiconducting compounds in the $2 \mathrm{H}$ phase.

The vibrational heat capacity at constant volume is calculated using

$$
C_{v}=k_{B} \int d \omega \rho(\omega) p(\omega, T)
$$

where $\rho$ is the phonon density of states, $p(x)=-x^{2} \partial f_{\mathrm{BE}} / \partial x$, $f_{B E}=1 /\left(e^{x}-1\right)$ being the Bose-Einstein distribution function, and $x=\hbar \omega / k_{B} T$. In Figs. 4 and 5 the vibrational heat capacities are plotted at $T=300 \mathrm{~K}, 500 \mathrm{~K}, 800 \mathrm{~K}$, and $1000 \mathrm{~K}$. At lower temperatures, the heat capacity is dominated by low frequency modes which possess low group velocity and larger phonon density of states. Therefore, heavier compounds, like $\mathrm{Hf}$ and $\mathrm{Zr}$ based structures, have higher heat
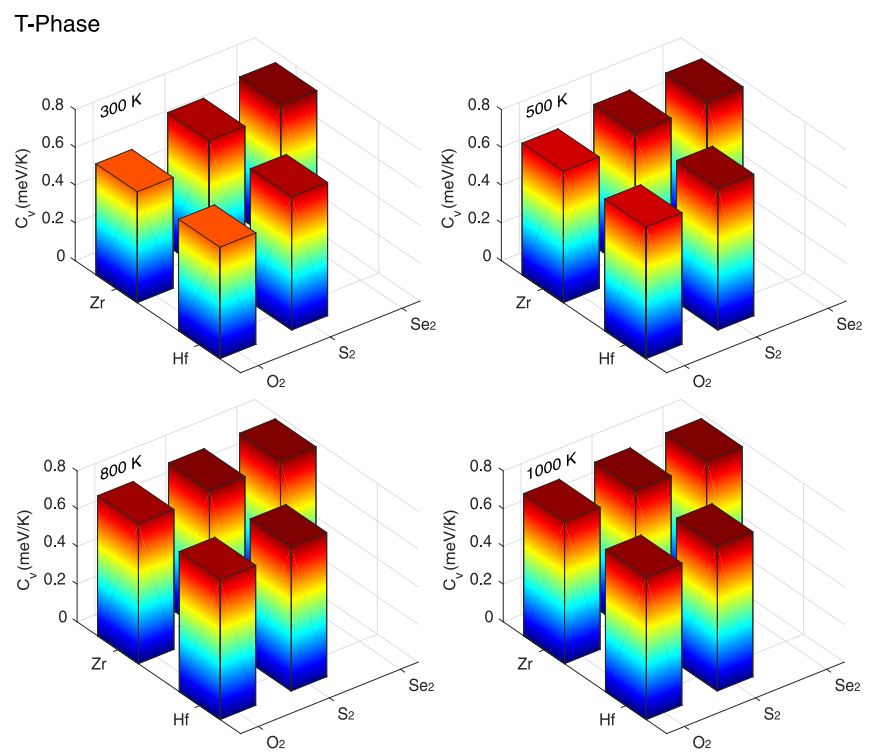

FIG. 5. Heat capacities at various temperatures from $300 \mathrm{~K}$ to $1000 \mathrm{~K}$ are shown for the semiconducting compounds in the $1 \mathrm{~T}$ phase. 
capacities at $300 \mathrm{~K}$. At increased temperatures, the differences in the $C_{v}$ tend to decrease. At $1000 \mathrm{~K}$, the function $p(x)$ is almost constant and equal to unity in the entire spectrum. The heat capacities approach the classical limit, which is proportional to the number of modes per unit cell.

\section{THERMOELECTRIC PROPERTIES}

According to the Mott formula [20,89,90]

$$
\left.S(T, \mu) \approx \frac{\pi^{2} k_{\mathrm{B}}^{2} T}{3 e} \frac{d \ln \tau(E)}{d E}\right|_{\mu},
$$

the logarithmic derivative of the electronic transmission determines the Seebeck coefficient at low temperatures. Namely, the abrupt changes in the transmission spectrum gives rise to large Seebeck coefficient and power factor. The structures studied in this work agree with this rule of thumb. The thermoelectric coefficients, $S, P$, and $Z T$, for various temperatures are tabulated in Table III. The chemical potential $(\mu)$ is chosen around the band edges where $Z T$ is maximized. The difference between $\mu$ at the valence band edge (conduction band edge) and the $\mu$ where $p$-type $Z T$ ( $n$-type $Z T$ ) is maximized is crucial for determining the optimal doping levels of the semiconductor (see Table S1) [93]. One observes that most of the $2 \mathrm{H}$ group-VIB TMD/TMOs have relatively low $Z T$ values compared to the $2 \mathrm{H}$ group-IVB TMD/TMOs. Notably, oxide compounds from group VIB show considerably weak TE performance due to their low atomic masses and hence high $\kappa_{p h}$. While $p$-type $Z T$ values of the group-VIB TMOs reach a maximum value around 0.11 at room temperature, the corresponding values for $n$-type $Z T$ can be as high as 0.16 . There are various theoretical studies on the TE properties of $\mathrm{MX}_{2}(\mathrm{M}=\mathrm{Mo}, \mathrm{W} ; \mathrm{X}=\mathrm{S}, \mathrm{Se})$ monolayers. In a previous work, $n$-type $Z T$ of the most studied $\mathrm{MoS}_{2}$ monolayer was predicted 0.04 by using the Boltzmann equation and equilibrium molecular dynamics (EMD) simulations [33]. Wickramaratne et al. obtained different $n$-type ZT values $(0.87 / 1.35)$ by adopting layer thickness dependent and constant $\kappa_{p h}$ values in diffusive regime calculations. In another work, reported $Z T$ values are overestimated compared to our findings for well-studied $\mathrm{MoS}_{2}, \mathrm{MoSe}_{2}, \mathrm{WS}_{2}$, and $\mathrm{WSe}_{2}$ in the frame of ballistic transport [30]. In addition, previously reported $p$ and $n$-type $Z T$ values $(0.58 / 0.25)$ in the ballistic regime are consistent with our results $(0.47 / 0.22)$ [34]. Also, there is an agreement on the results of Huang et al. that $p$-type $Z T$ of $\mathrm{MoS}_{2}$ at room temperature and $n$-type $Z T$ of $\mathrm{WSe}_{2}$ at high temperatures are found to be higher than those of the $\mathrm{MoSe}_{2}$ and $\mathrm{WS}_{2}$ [34].

Among all investigated compounds, $\mathrm{ZrSe}_{2}, \mathrm{HfS}_{2}$, and $\mathrm{HfSe}_{2}$ are dynamically stable in both $2 \mathrm{H}$ and $1 \mathrm{~T}$ phases.

TABLE III. $p$ - and $n$-type Seebeck coefficient $(S)$, power factor $(P)$, and thermoelectric figure of merit $(Z T)$ at different temperatures based on PBE calculations.

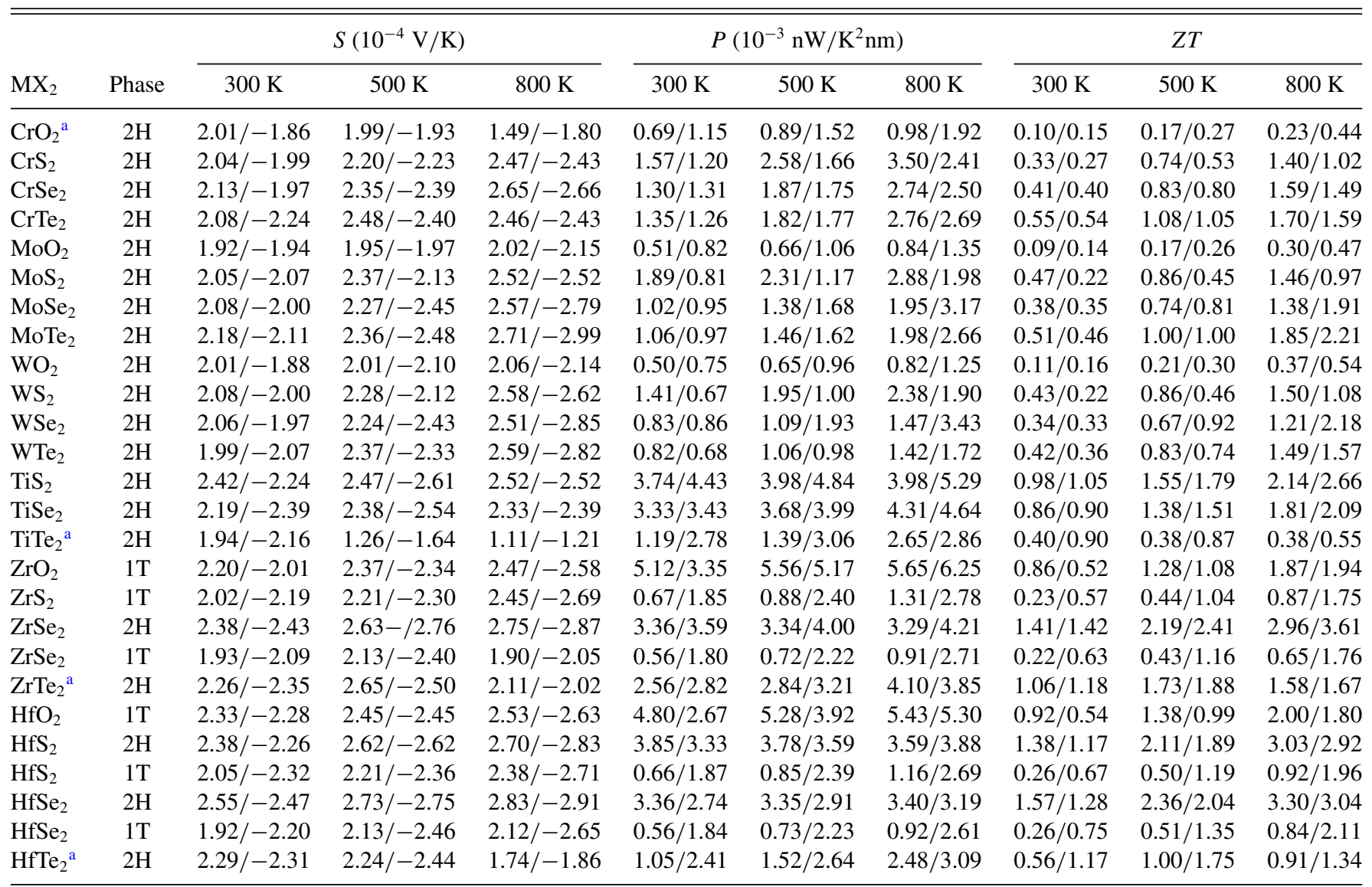

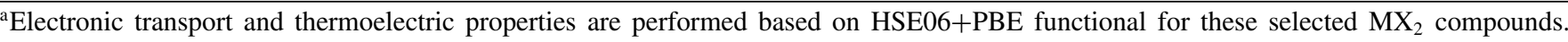
$\mathrm{p} / \mathrm{n}$-type ZT values for various temperatures are listed in Table IV. In addition $\tau_{e l}(E), S, P F$, and ZT are demonstrated in Fig. S3 [93]. 
TABLE IV. $p$ - and $n$-type $Z T$ values at different temperatures based on the HSE06 calculations.

\begin{tabular}{lcccc}
\hline \hline & & \multicolumn{3}{c}{$Z T(p$ - $/ n$-type $)$} \\
\cline { 3 - 5 } $\mathrm{MX}_{2}$ & Phase & $300 \mathrm{~K}$ & $500 \mathrm{~K}$ & $800 \mathrm{~K}$ \\
\hline $\mathrm{CrO}_{2}$ & $2 \mathrm{H}$ & $0.09 / 0.13$ & $0.17 / 0.23$ & $0.30 / 0.42$ \\
$\mathrm{TiTe}_{2}$ & $2 \mathrm{H}$ & $0.47 / 0.81$ & $0.90 / 1.37$ & $1.70 / 2.18$ \\
$\mathrm{ZrTe}_{2}$ & $2 \mathrm{H}$ & $0.80 / 1.08$ & $1.49 / 1.74$ & $2.47 / 2.67$ \\
$\mathrm{HfTe}_{2}$ & $2 \mathrm{H}$ & $0.52 / 1.03$ & $1.00 / 1.71$ & $1.76 / 2.62$ \\
$\mathrm{TiS}_{2}$ & $1 \mathrm{~T}$ & $0.11 / 0.37$ & $0.21 / 0.70$ & $0.38 / 1.21$ \\
\hline \hline
\end{tabular}

We predict substantial differences in their TE performances. Both $n$-type and $p$-type $Z T$ values of the $2 \mathrm{H}$ phases are much larger than those of the $1 \mathrm{~T}$ phase, and $\kappa_{\mathrm{ph}}$ of the $1 \mathrm{~T}$ phases are always slightly higher than the $2 \mathrm{H}$ phases (see Table II). The underlying reason lies mostly in their electronic band structures. The frontier bands in the $2 \mathrm{H}$ phase are less dispersive than in the 1T phase. The valence band maximum is almost flat, which leads to sharp changes in the DOS and the electronic transmission spectrum and give rise to enhanced $S$, $P$, and $Z T$. As a result, the $p$-type $Z T$ values of the $2 \mathrm{H}$ phases are five to six times higher than those of the $1 \mathrm{~T}$ phases. In the $n$-type $Z T$, the difference is not as dramatic as in the $p$-type $Z T$, but those of $2 \mathrm{H}$ phases are considerably larger again. $1 \mathrm{~T}$ phases of $\mathrm{ZrSe}_{2}, \mathrm{HfSe}_{2}$, and $\mathrm{HfS}_{2}$ were previously predicted to have promising $Z T$ values, when phonon scatterings are taken into account $[53,56]$.

In order to quantify the role of $\kappa_{\mathrm{ph}}$ on $Z T$, we study their correlation from the available data. The $p$-type $Z T$ for $2 \mathrm{H}$ compounds is inversely correlated with $\kappa_{\mathrm{ph}}$ as $55 \%, 60 \%$, and $59 \%$ at $300 \mathrm{~K}, 500 \mathrm{~K}$, and $800 \mathrm{~K}$, respectively. On the other hand, inverse correlation between $n$-type $Z T$ and $\kappa_{\mathrm{ph}}$ is slightly larger than that of $p$-type $Z T$. Inverse correlation values of $57 \%, 62 \%$, and $61 \%$ are obtained for the same temperatures. These illustrate the role of $\kappa_{\mathrm{ph}}$ in determining the TE performance of the crystals considered.

The $2 \mathrm{H}$ phases of $\mathrm{ZrSe}_{2}, \mathrm{ZrTe}_{2} \mathrm{HfS}_{2}$, and $\mathrm{HfSe}_{2}$ provide $Z T$ values larger than 1 for both $n$ - and $p$-type carriers at room temperature. The electronic transmission, Seebeck coefficient, power factor, and TE figure of merit of these compounds are presented in Fig. 6. Although $2 \mathrm{H}-\mathrm{ZrSe}_{2}$ and $2 \mathrm{H}-\mathrm{ZrTe}_{2}$ have almost the same thermal conductance values, $\mathrm{ZrTe}_{2}$ has the lowest $Z T$ compared to other promising compounds due to relatively smooth transmission spectra at the valence band edge. It is also observed that PBE results yield a decreasing $Z T$ for $Z \mathrm{ZTe}_{2}$ above $500 \mathrm{~K}$. This stems from the fact that the band gap of $\mathrm{ZrTe}_{2}$ as predicted from PBE is not sufficient to support an efficient TE response at high temperatures. We use hybrid functionals to correct the calculated band gap, which will be discussed separately below. Abrupt changes in the transmission spectra are observed at the valence band edges of $\mathrm{ZrSe}_{2}, \mathrm{HfS}_{2}$, and $\mathrm{HfSe}_{2}$ (see Fig. 6).

Altough $\kappa_{\mathrm{ph}}$ of $2 \mathrm{H}-\mathrm{HfTe} \mathrm{T}_{2}$ is lower than that of $2 \mathrm{H}-\mathrm{HfSe} \mathrm{e}_{2}$, its $p$-type $Z T$ is much lower than that of $2 \mathrm{H}-\mathrm{HfSe}_{2}$, which is found to have the highest $p$-type $Z T$ value (1.57) at room temperature. The lower $Z T$ value of $2 \mathrm{H}-\mathrm{HfTe} \mathrm{Te}_{2}$ is because of its electronic transmission being smoother than that of $2 \mathrm{H}-\mathrm{HfSe}_{2}$. In the case of $n$-type $Z T$, in addition to these
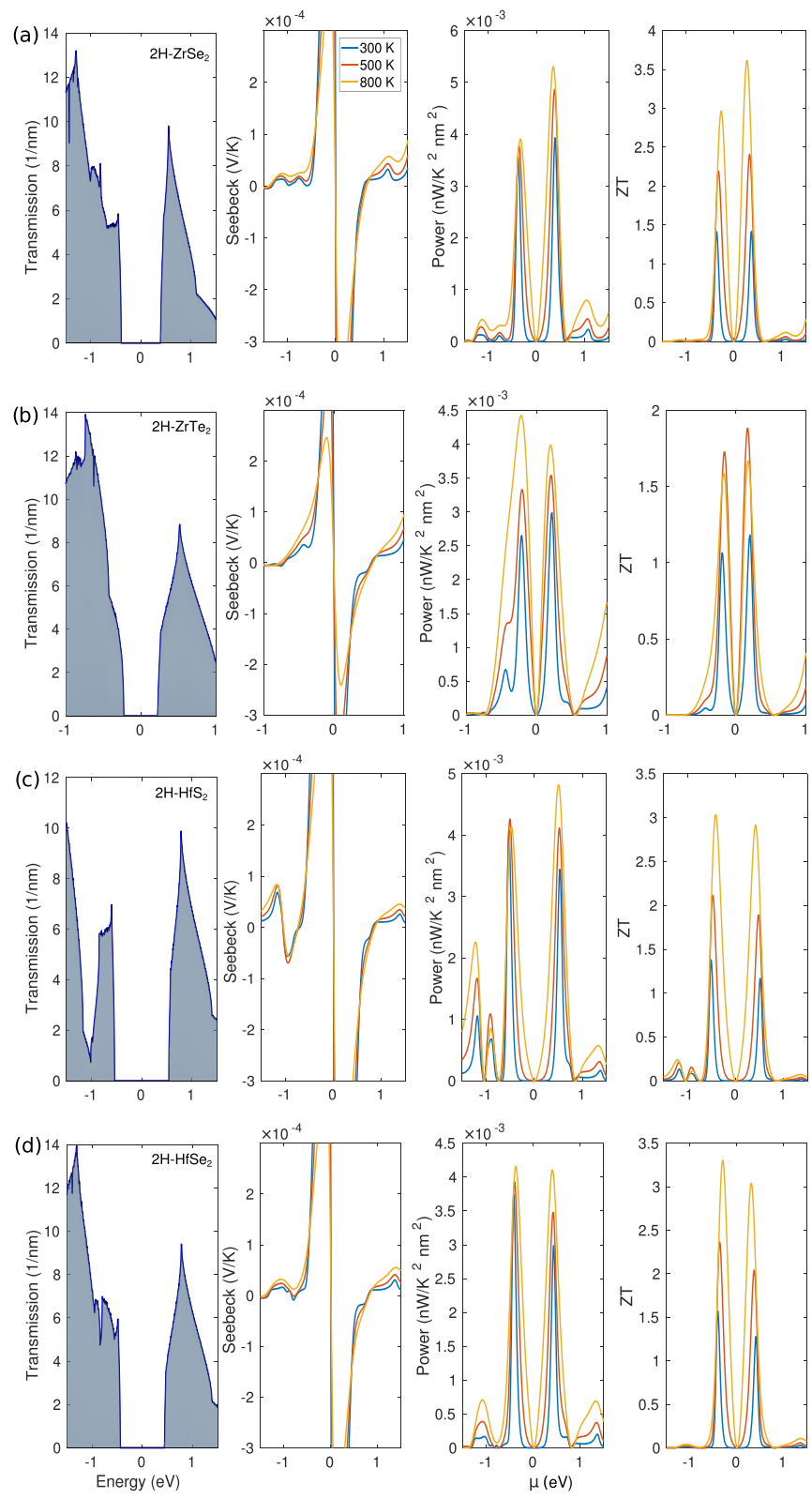

FIG. 6. Electronic transmission, Seebeck coefficient, power factor, and thermoelectric figure of merit are plotted around the Fermi level for $2 \mathrm{H}-\mathrm{ZrSe}_{2}, 2 \mathrm{H}-\mathrm{ZrTe}_{2}, 2 \mathrm{H}-\mathrm{HfS}_{2}$, and $2 \mathrm{H}-\mathrm{HfSe}{ }_{2}$.

four TMDs, for $2 \mathrm{H}-\mathrm{HfTe} 2$ and $2 \mathrm{H}-\mathrm{TiS} 2$, it exceeds 1 at room temperature. It is worth mentioning that a remarkably high $p$-type power factor is achieved for the $1 \mathrm{~T}-\mathrm{ZrO}_{2}$ and $1 \mathrm{~T}-\mathrm{HfO}_{2}$ but their lattice thermal conductances are higher than those of $2 \mathrm{H}-\mathrm{ZrSe}_{2}, 2 \mathrm{H}-\mathrm{ZrTe}_{2}, 2 \mathrm{H}-\mathrm{HfS}_{2}$, and $2 \mathrm{H}-\mathrm{HfSe}_{2}$ by about a factor of 2 or 3 , thus their $Z T$ values remain under 1 at $300 \mathrm{~K}$. In principle, TE response of these oxides can be enhanced by reducing $\kappa_{p h}$ with phonon engineering.

Seebeck coefficient is reduced with simultaneous contribution of $p$ - and $n$-type carriers. Accordingly, obtaining accurate $S, P F$, and $Z T$ values will mostly depend on electronic band gap of material, especially at higher temperatures. If the band gap of the material is smaller than about $10 \mathrm{k}_{B} T, S$ is suppressed with increasing temperature as in the cases of 


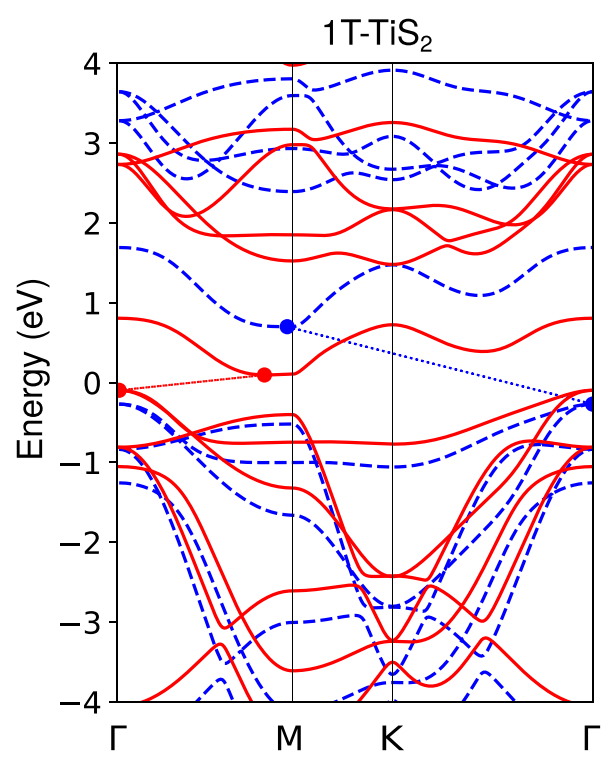

FIG. 7. Electronic band structure of $1 \mathrm{~T}-\mathrm{TiS}_{2}$ with PBE (solid red) and hybrid HSE06 (dashed blue) functionals. Transition from semimetallic to semiconducting phase occurs with the hybrid functional.

$2 \mathrm{H}-\mathrm{CrO}_{2}, 2 \mathrm{H}-\mathrm{HfTe}_{2}, 2 \mathrm{H}-\mathrm{TiTe}_{2}$, and $2 \mathrm{H}-\mathrm{ZrTe}_{2}$. The $E_{\mathrm{g}}^{\mathrm{PBE}}$ is $0.36 \mathrm{eV}(0.45 \mathrm{eV})$ for $2 \mathrm{H}-\mathrm{HfTe}_{2}\left(2 \mathrm{H}-\mathrm{ZrTe}_{2}\right)$, and $S$ is suppressed when temperature is above $300 \mathrm{~K}$ (500 K). Suppression of $S$ reduces $Z T$ when $T$ is above $500 \mathrm{~K}$ for $2 \mathrm{H}-\mathrm{HfTe}_{2}$, because the increase in $G$ compensates the decrease in $S$ at lower temperatures. For $2 \mathrm{H}-\mathrm{CrO}_{2}$, a similar trend in $S$ is observed, however $Z T$ is not suppressed at higher temperatures because $G$ increases with $T$. $2 \mathrm{H}-\mathrm{TiTe}_{2}$ has the narrowest $E_{\mathrm{g}}^{\mathrm{HSE}}(0.19 \mathrm{eV})$ among the investigated TMDs. Therefore, the decrease in $S$ and $Z T$ appear above room temperature. $\tau_{e l}(E)$, $S, P F$, and $Z T$ calculated from hybrid-functional-corrected band gaps are demonstrated in the Supplemental Material (see Fig. S3) [93]. Band gap correction using hybrid functionals results in better $Z T$ values for these $\mathrm{MX}_{2}$ compounds as seen in Table IV.
Besides the semiconducting TMD/TMOs, TE properties of semimetallic $1 \mathrm{~T}-\mathrm{TiS}_{2}$ is also investigated. $1 \mathrm{~T}-\mathrm{TiS}_{2}$ is more stable with a $E_{\mathrm{coh}}=5.31 \mathrm{eV}$, which is higher than its $2 \mathrm{H}$ phase for about $0.14 \mathrm{eV}$ [91]. HSE06 correction exhibits a transition from semimetallic to semiconducting behavior as shown in Fig. 7 with a band gap of $0.62 \mathrm{eV}$, in agreement with previous results [92]. It is clearly seen that $1 \mathrm{~T}-\mathrm{TiS}_{2}$ does not achieve a high value of $p$-type $Z T$, but $n$-type $Z T$ exceeds 1 when temperature reaches $800 \mathrm{~K}$ (see Table IV and Fig. S3) [93].

We note that inclusion of spin-orbit coupling (SOC) should be expected to give rise to quantitative changes in the thermoelectric coefficients of the structures. Lifting the spin degeneracy, SOC can be expected to reduce the TE efficiency for structures where the SOC is strong.

\section{CONCLUSION}

We have investigated structural, electronic, vibrational, as well as ballistic transport and thermoelectric properties of a large family of TMDs/TMOs by using a combination of $a b$ initio and Landauer-Büttiker formalisms. We have identified promising thermoelectric materials which possess high $Z T$ values close to or above 1 at room temperature. In particular, high $p$-type and $n$-type TE figure of merit are found for $2 \mathrm{H}-\mathrm{HfSe}_{2}$ and $2 \mathrm{H}-\mathrm{ZrSe}_{2}$, respectively. Moreover, our calculations reveal that two TMO monolayers, $1 \mathrm{~T}-\mathrm{ZrO}_{2}$ and $1 \mathrm{~T}-\mathrm{HfO}_{2}$, can be promising $p$-type thermoelectric candidates at room temperature.

\section{ACKNOWLEDGMENTS}

G.Ö., R.T.S., and H.S. acknowledge support from Scientific and Technological Research Council of Turkey (TUBITAK) Grant No. 117F131. C.S. acknowledges the support from BAGEP Award of the Science Academy. Part of the numerical calculations are carried out at TUBITAK ULAKBIM High Performance and Grid Computing Center.
[1] B. Poudel, Q. Hao, Y. Ma, Y. Lan, A. Minnich, B. Yu, X. Yan, D. Wang, A. Muto, D. Vashaee, X. Chen, J. Liu, M. S. Dresselhaus, G. Chen, and Z. Ren, Science 320, 634 (2008).

[2] L. D. Hicks and M. S. Dresselhaus, Phys. Rev. B 47, 12727 (1993).

[3] L. D. Hicks and M. S. Dresselhaus, Phys. Rev. B 47, 16631 (1993).

[4] G. D. Mahan and H. B. Lyon, J. Appl. Phys. 76, 1899 (1994).

[5] L. D. Hicks, T. C. Harman, X. Sun, and M. S. Dresselhaus, Phys. Rev. B 53, R10493 (1996).

[6] D. A. Broido and T. L. Reinecke, Appl. Phys. Lett. 67, 100 (1995).

[7] M. Dresselhaus, G. Chen, M. Tang, R. Yang, H. Lee, D. Wang, Z. Ren, J.-P. Fleurial, and P. Gogna, Adv. Mater. 19, 1043 (2007).

[8] T. A. Amollo, G. T. Mola, M. S. K. Kirui, and V. O. Nyamori, Crit. Rev. Solid State Mater. Sci. 43, 133 (2018).
[9] P. Dollfus, V. H. Nguyen, and J. Saint-Martin, J. Phys.: Condens. Matter 27, 133204 (2015).

[10] Y. Xu, Z. Li, and W. Duan, Small 10, 2182 (2014).

[11] Y. M. Zuev, W. Chang, and P. Kim, Phys. Rev. Lett. 102, 096807 (2009).

[12] F. Ghahari, H.-Y. Xie, T. Taniguchi, K. Watanabe, M. S. Foster, and P. Kim, Phys. Rev. Lett. 116, 136802 (2016).

[13] A. A. Balandin, Nat. Mater. 10, 569 (2011).

[14] J.-W. Jiang, J.-S. Wang, and B. Li, Phys. Rev. B 79, 205418 (2009).

[15] Z. Wang, R. Xie, C. T. Bui, D. Liu, X. Ni, B. Li, and J. T. L. Thong, Nano Lett. 11, 113 (2011).

[16] Y. Anno, K. Takei, S. Akita, and T. Arie, Adv. Electron. Mater. 1, 1500175 (2015).

[17] Y. Anno, Y. Imakita, K. Takei, S. Akita, and T. Arie, 2D Materials 4, 025019 (2017). 
[18] R. D'Souza and S. Mukherjee, J. Appl. Phys. 124, 124301 (2018).

[19] D. Dragoman and M. Dragoman, Appl. Phys. Lett. 91, 203116 (2007).

[20] T. Gunst, T. Markussen, A.-P. Jauho, and M. Brandbyge, Phys. Rev. B 84, 155449 (2011).

[21] H. Sevinçli, C. Sevik, T. Çağin, and G. Cuniberti, Sci. Rep. 3, 1228 (2013).

[22] H. Sevinçli and G. Cuniberti, Phys. Rev. B 81, 113401 (2010).

[23] L. M. Sandonas, H. Sevinçli, R. Gutierrez, and G. Cuniberti, Adv. Sci. 5, 1700365 (2018).

[24] F. Mazzamuto, V. Hung Nguyen, Y. Apertet, C. Caër, C. Chassat, J. Saint-Martin, and P. Dollfus, Phys. Rev. B 83, 235426 (2011).

[25] Y. Ouyang and J. Guo, Appl. Phys. Lett. 94, 263107 (2009).

[26] K. Yang, Y. Chen, R. D’Agosta, Y. Xie, J. Zhong, and A. Rubio, Phys. Rev. B 86, 045425 (2012).

[27] C. Ataca, H. Sahin, and S. Ciraci, J. Phys. Chem. C 116, 8983 (2012).

[28] C. Adessi, S. Thebaud, R. Bouzerar, and G. Bouzerar, J. Phys. Chem. C 121, 12577 (2017).

[29] B. Ouyang, S. Chen, Y. Jing, T. Wei, S. Xiong, and D. Donadio, J. Materiomics 4, 329 (2018).

[30] K.-X. Chen, X.-M. Wang, D.-C. Mo, and S.-S. Lyu, J. Phys. Chem. C 119, 26706 (2015).

[31] H. Babaei, J. M. Khodadadi, and S. Sinha, Appl. Phys. Lett. 105, 193901 (2014).

[32] K. Nakamura, Jpn. J. Appl. Phys. 57, 06 HE04 (2018).

[33] Z. Jin, Q. Liao, H. Fang, Z. Liu, W. Liu, Z. Ding, T. Luo, and N. Yang, Sci. Rep. 5, 18342 (2015).

[34] W. Huang, H. Da, and G. Liang, J. Appl. Phys. 113, 104304 (2013).

[35] G. Zhang and Y.-W. Zhang, J. Mater. Chem. C 5, 7684 (2017).

[36] S. Kumar and U. Schwingenschlögl, Chem. Mater. 27, 1278 (2015).

[37] A. Arab and Q. Li, Sci. Rep. 5, 13706 (2015).

[38] D. Wickramaratne, F. Zahid, and R. K. Lake, J. Chem. Phys. 140, 124710 (2014).

[39] M. Kayyalha, J. Maassen, M. Lundstrom, L. Shi, and Y. P. Chen, J. Appl. Phys. 120, 134305 (2016).

[40] K. Hippalgaonkar, Y. Wang, Y. Ye, D. Y. Qiu, H. Zhu, Y. Wang, J. Moore, S. G. Louie, and X. Zhang, Phys. Rev. B 95, 115407 (2017).

[41] W. Huang, X. Luo, C. K. Gan, S. Y. Quek, and G. Liang, Phys. Chem. Chem. Phys. 16, 10866 (2014).

[42] R.-N. Wang, G.-Y. Dong, S.-F. Wang, G.-S. Fu, and J.-L. Wang, Phys. Chem. Chem. Phys. 19, 5797 (2017).

[43] Y. Ouyang, Y. Xie, Z. Zhang, Q. Peng, and Y. Chen, J. Appl. Phys. 120, 235109 (2016).

[44] Z. Zhang, Y. Xie, Q. Peng, and Y. Chen, Sci. Rep. 6, 21639 (2016).

[45] T.-m. Wu, R.-x. Xu, X. Zheng, and W. Zhuang, Chin. J. Chem. Phys. 29, 445 (2016).

[46] Y.-Y. Liu, Y.-J. Zeng, P.-Z. Jia, X.-H. Cao, X. Jiang, and K.-Q. Chen, J. Phys.: Condens. Matter 30, 275701 (2018).

[47] J. Hong, C. Lee, J.-S. Park, and J. H. Shim, Phys. Rev. B 93, 035445 (2016).

[48] W. Shen, D. Zou, G. Nie, and Y. Xu, Chin. Phys. B 26, 117202 (2017).
[49] Dimple, N. Jena, and A. D. Sarkar, J. Phys.: Condens. Matter 29, 225501 (2017).

[50] S.-D. Guo, Comput. Mater. Sci. 123, 8 (2016).

[51] G. Zhang and Y.-W. Zhang, Mech. Mater. 91, 382 (2015).

[52] H. Y. Lv, W. J. Lu, D. F. Shao, H. Y. Lu, and Y. P. Sun, J. Mater. Chem. C 4, 4538 (2016).

[53] G. Ding, G. Y. Gao, Z. Huang, W. Zhang, and K. Yao, Nanotechnology 27, 375703 (2016).

[54] S.-D. Guo and J.-L. Wang, Semicond. Sci. Technol. 31, 095011 (2016).

[55] Y.-X. Zhen, M. Yang, H. Zhang, G.-S. Fu, J.-L. Wang, S.-F. Wang, and R.-N. Wang, Science Bulletin 62, 1530 (2017).

[56] D. Qin, X.-J. Ge, G.-q. Ding, G.-y. Gao, and J.-T. Lü, RSC Adv. 7, 47243 (2017).

[57] H. Y. Lv, W. J. Lu, X. Luo, H. Y. Lu, X. B. Zhu, and Y. P. Sun, arXiv:1608.05464.

[58] G. Kresse and J. Furthmüller, Phys. Rev. B 54, 11169 (1996).

[59] G. Kresse and D. Joubert, Phys. Rev. B 59, 1758 (1999).

[60] J. P. Perdew, K. Burke, and M. Ernzerhof, Phys. Rev. Lett. 77, 3865 (1996).

[61] H. J. Monkhorst and J. D. Pack, Phys. Rev. B 13, 5188 (1976).

[62] A. V. Krukau, O. A. Vydrov, A. F. Izmaylov, and G. E. Scuseria, J. Chem. Phys. 125, 224106 (2006).

[63] S. Baroni, S. de Gironcoli, A. Dal Corso, and P. Giannozzi, Rev. Mod. Phys. 73, 515 (2001).

[64] A. Togo and I. Tanaka, Scr. Mater. 108, 1 (2015).

[65] G. Henkelman, A. Arnaldsson, and H. Jónsson, Comput. Mater. Sci. 36, 354 (2006).

[66] W. Callister and D. Rethwisch, Materials Science and Engineering: An Introduction, 9th Edition: Ninth Edition (John Wiley and Sons, Incorporated, New York, 2013).

[67] U. Sivan and Y. Imry, Phys. Rev. B 33, 551 (1986).

[68] K. Esfarjani, M. Zebarjadi, and Y. Kawazoe, Phys. Rev. B 73, 085406 (2006).

[69] L. G. C. Rego and G. Kirczenow, Phys. Rev. Lett. 81, 232 (1998).

[70] H. Sevinçli, S. Roche, G. Cuniberti, M. Brandbyge, R. Gutierrez, and L. M. Sandonas, J. Phys.: Condens. Matter 31, 273003 (2019).

[71] F. A. Rasmussen and K. S. Thygesen, J. Phys. Chem. C 119, 13169 (2015).

[72] C. Gong, H. Zhang, W. Wang, L. Colombo, R. M. Wallace, and K. Cho, Appl. Phys. Lett. 103, 053513 (2013).

[73] P. Tsipas, D. Tsoutsou, S. Fragkos, R. Sant, C. Alvarez, H. Okuno, G. Renaud, R. Alcotte, T. Baron, and A. Dimoulas, ACS Nano 12, 1696 (2018).

[74] S. Aminalragia-Giamini, J. Marquez-Velasco, P. Tsipas, D. Tsoutsou, G. Renaud, and A. Dimoulas, 2D Materials 4, 015001 (2017).

[75] M. J. Mleczko, C. Zhang, H. R. Lee, H.-H. Kuo, B. MagyariKöpe, R. G. Moore, Z.-X. Shen, I. R. Fisher, Y. Nishi, and E. Pop, Sci. Adv. 3, e1700481 (2017).

[76] C. Yan, C. Gong, P. Wangyang, J. Chu, K. Hu, C. Li, X. Wang, X. Du, T. Zhai, Y. Li, and J. Xiong, Adv. Funct. Mater. 28, 1803305 (2018).

[77] G. Mahan, B. Sales, and J. Sharp, Phys. Today 50(3), 42 (1997).

[78] J. O. Sofo and G. D. Mahan, Phys. Rev. B 49, 4565 (1994). 
[79] R. P. Chasmar and R. Stratton, J. Electron. Control 7, 52 (1959).

[80] G. S. Nolas, J. Sharp, and H. J. Goldsmid, Thermoelectrics: Basic Principles and New Materials Development (SpringerVerlag, Berlin, Heidelberg, 2001).

[81] A. M. Dehkordi, M. Zebarjadi, J. He, and T. M. Tritt, Mater. Sci. Eng., R 97, 1 (2015).

[82] B. Singh, C.-H. Hsu, W.-F. Tsai, V. M. Pereira, and H. Lin, Phys. Rev. B 95, 245136 (2017).

[83] D. L. Duong, M. Burghard, and J. C. Schön, Phys. Rev. B 92, 245131 (2015).

[84] M. J. Wei, W. J. Lu, R. C. Xiao, H. Y. Lv, P. Tong, W. H. Song, and Y. P. Sun, Phys. Rev. B 96, 165404 (2017).

[85] C. Chen, B. Singh, H. Lin, and V. M. Pereira, Phys. Rev. Lett. 121, 226602 (2018).
[86] J.-A. Yan, M. A. D. Cruz, B. Cook, and K. Varga, Sci. Rep. 5, 16646 (2015).

[87] Z. Zhang, Y. Xie, Y. Ouyang, and Y. Chen, Int. J. Heat Mass Transf. 108, 417 (2017).

[88] Y. Cai, J. Lan, G. Zhang, and Y.-W. Zhang, Phys. Rev. B 89, 035438 (2014).

[89] M. Cutler and N. F. Mott, Phys. Rev. 181, 1336 (1969).

[90] G. D. Guttman, E. Ben-Jacob, and D. J. Bergman, Phys. Rev. B 51, 17758 (1995).

[91] C. Xu, P. A. Brown, and K. L. Shuford, RSC Adv. 5, 83876 (2015).

[92] A. Samad, A. Shafique, and Y.-H. Shin, Nanotechnology 28, 175401 (2017).

[93] See Supplemental Material at http://link.aps.org/supplemental/ 10.1103/PhysRevB.100.085415 for further figures and tables. 